\title{
«MA INFINE NELLA VITA TUTTO È MORTE!» COSA CI RACCONTA IL TROVATORE?
}

\author{
FABRIZIO DELLA SETA $(*)$ \\ A Franco Piperno per i suoi sessant'anni e per quaranta di amicizia \\ «The music that makes one want to cry», he called it, \\ and he always wanted it played at a volume that jarred my eardrums \\ and severely taxed the capacity of the tape recorder.
}

RIASSUNTO - Opera da sempre tra le più amate dal grande pubblico, Il trovatore è oggetto di una notevole letteratura critica, che però raramente si è interrogata sul suo 'significato', nella sottintesa convinzione che, proprio per la sua natura di melodramma eminentemente 'popolare', essa non trasmetta nessun particolare messaggio di ordine ideologico, sociale o semplicemente umano. Un luogo comune sul libretto è la sua oscurità: gran parte della vicenda rimanda a un antefatto che non viene presentato sulla scena; non è chiaro quale sia il numero e la successione degli scontri tra Manrico e il Conte di Luna; la vera identità di Manrico resta elusiva. Piuttosto che condannare l'assurdità del Trovatore, conviene assumerla come una sua caratteristica strutturale. La rievocazione di fatti del passato e la scarsa chiarezza circa l'ordine cronologico di essi richiamano una struttura temporale tipica delle narrazioni tradizionali, che ignorano distinzione tra prima e dopo e rapporto di causalità. L'identità della persona non è un problema per la logica delle emozioni, che ignora il principio di non contraddizione, secondo la teoria freudiana dell'inconscio rielaborata da Ignacio Matte Blanco e applicata alla teoria della letteratura da Francesco Orlando, il quale ha mostrato i procedimenti formali grazie ai quali l'opera d'arte realizza il ritorno di contenuti repressi, perché socialmente censurati, attenuandoli e mascherandoli per renderli accettabili. In questa prospettiva, il saggio sostiene che nel Trovatore poeta e

(*) Istituto Lombardo Accademia di Scienze e Lettere, Università degli Studi di Pavia.

1 Così, sul Trovatore, il capo Utku Inuttiaq, come riferito da J.L. Briggs, Never in anger: portrait of an Eskimo family, Cambridge, Mass., Harvard University Press, 1970, 154. 
musicista hanno dato forma scenico-musicale a un'idea centrale dell'immaginario occidentale e particolarmente romantico, che Freud ha ripensato nel suo pensiero più maturo: la compresenza, nella natura umana, di un intreccio inestricabile tra pulsione di vita e pulsione di morte (in termini mitologici tra Eros e Thanatos). Esse si incarnano in proporzioni variabili in tutti e quattro i protagonisti, secondo una progressione che evidenzia il predominio assoluto della pulsione di morte in Leonora. Questa tesi è verificata con un'analisi che prende in considerazione le componenti verbali del messaggio drammatico - anche attraverso un confronto col dramma spagnolo da cui l'opera è tratta - e che interpreta la funzione delle componenti musicali, particolarmente della virtuosistica scrittura vocale di Leonora. Tale interpretazione implica una revisione della posizione comunemente attribuita al Trovatore nel percorso creativo del compositore: non che essere opera retrospettiva, proprio in virtù dell'impiego sistematico di forme e di stili vocali desueti essa apre una nuova fase di tale percorso, che conduce dal tenace attaccamento alla vita e all'amore degli eroi delle opere giovanili, anche quando sconfitti, alla disillusione e alla ricerca di «pace» e «oblio» che percorrono la produzione matura e tarda di Verdi.

ABSTRACT - One of the best loved of Verdi's operas, Il trovatore has accumulated a considerable critical literature, and yet commentators have rarely enquired into its 'meaning', apparently out of the conviction that, precisely because it is a pre-eminently 'popular' work, it has no particular ideological, social or indeed human message to convey. One received opinion concerning the opera is that it has an obscure libretto: much of the story refers to a prior event that is not presented on stage. We know that Manrico and the Conte di Luna have clashed, but we don't know how often, or anything about the dynamic of this confrontation, while Manrico's true identity remains elusive. However, rather than condemning the intrinsic absurdity of Il Trovatore, we would do better to view this as a structural characteristic. The evocation of facts from the past and the lack of clarity concerning their chronological order allude to a temporal structure typical of traditional narrations in which the distinction between before and after and the relationship of cause and effect are ignored. Personal identity is not a problem for the logic of the emotions, which has no knowledge of the principle of non-contradiction, according to Freud's theory of the unconscious as developed by Ignacio Matte Blanco. In applying this principle to the theory of literature, Francesco Orlando revealed the formal procedures by which the work of art achieves the reinstatement of contents that have been repressed on account of social censorship, attenuating them and disguising them to make them acceptable. This essay argues that in Il Trovatore poet and musician have given scenic and musical form to an idea that is central in Western, and particularly Romantic, imagination, one which Freud reformulated in his maturity: the presence, in human nature, of an inextricable alliance of the urge to live and the death drive (in mythological terms, of Eros and Thanatos). These two impulses are incarnated in variable proportions in all four protagonists, with a progression that culminates in the absolute predominance of the death wish in Leonora. This thesis is verified by means of an analysis focusing on both the verbal components of the drama's message - including a comparison with the Spanish play on which the opera is based and the function of the musical components, in particular the virtuoso vocal writing assigned to Leonora. This interpretation requires a revision of the position usually attributed to Il Trovatore in Verdi's creative development as a retrospective work. Precisely 
the systematic use of old-fashioned vocal forms and styles denotes a new phase in the composer's parabola, as the tenacious adhesion to life and love of the heroes of the youthful operas, even when defeated, gives way to the disillusionment and quest for «peace» and «forgetfulness» that characterise Verdi's mature and last works.

Qualche tempo fa mi è capitato di scrivere che Il trovatore è l'opera «più difficile dell'intero canone». ${ }^{2}$ Non pensavo certo a una difficoltà di afferrarne le idee musicali e di seguire il loro rincorrersi: tutti sanno che è una delle opere in assoluto più amate e che contiene alcune delle melodie più orecchiabili e ripetute dell'intero repertorio melodrammatico; mi riferivo piuttosto alla difficoltà di coglierne un messaggio, un significato. Di cosa 'ci parla' Il trovatore? A qualcuno sembrerà un non problema, ma non lo è per chi è convinto che ogni grande opera d'arte c'interroga sui fondamenti dell'esistenza umana. Tuttavia, mentre nessuno, per esempio, ha dubbi sul fatto che Rigoletto e La traviata ci parlano d'ingiustizia e giustizia, di oppressione e riscatto, di egoismo e sacrificio, quando si cerca di rispondere a quella domanda a proposito del Trovatore, si ha l'impressione di voler afferrare con le mani un polipo i cui tentacoli sfuggono da ogni parte. Ciò è molto evidente quando ripercorro la letteratura critica sull'opera, che pure ha una consistenza notevole ed è stata alimentata anche in anni recenti da studiosi di primo piano: $:^{3}$ ciascuno di loro ha detto sul Trovatore cose giuste e importanti, eppure resto con un'impressione di non detto, di prospettiva parziale. Mi sembra che ciò non accada con nessun'altra opera di Verdi o di altro autore.

2 F. Della Seta, «...non senza pazzia». Prospettive sul teatro musicale, Roma, Carocci, 2008, 15.

3 Si vedano in particolare: P. Petrobelli, Per un'esegesi della struttura drammatica del "Trovatore" (1974), in Id., La musica nel teatro. Saggi su Verdi e altri compositori, Torino, EDT, 1998, 107-20; J. Budden, Le opere di Verdi, 2, Dal "Trovatore" alla "Forza del destino", Torino, EDT/Musica, 1986, 61-124 (ed. or.: The operas of Verdi, 2, From "il trovatore" to "La forza del Destino, London, Cassell, 1978); W. Drabkin, Characters, key relations and tonal structure in "Il trovatore", Music analysis 1, 1982, 143-53; R. Parker, The dramatic structure of "Il trovatore", ivi, 155-67; A. Gerhard, Dalla fatalità all'ossessione: "Il trovatore" fra mélodrame parigino e opera moderna, Studi verdiani 10, 1994-5, 61-6; J. Hepokoski, Ottocento opera as cultural drama: generic mixtures in "Il trovatore", 
È utile riepilogare brevemente le principali linee critiche che si sono avvicendate nelle storia della ricezione del Trovatore. In origine, diciamo all'inizio del Novecento, il problema di un'interpretazione dell'opera 'come dramma' non si poneva neppure: in un clima ancora dominato dal wagnerismo, essa sembrava l'emblema di una forma d'intrattenimento popolare non degna di essere presa in considerazione come 'grande arte', un teatro di marionette reso seducente da melodie di facile effetto. Questa valutazione negativa, sempre più insostenibile alla luce non solo del perdurante favore goduto dall'opera presso vasti pubblici, ma anche dell'irresistibile attrazione che ha sempre esercitato su raffinati intellettuali, ${ }^{4}$ fu notoriamente ribaltata - ma conservandone i presupposti - negli anni Venti dalla Verdi-Renaissance tedesca ${ }^{5}$ e, in Italia, da Bruno Barilli, secondo cui Verdi «raggiunse con una immediatezza tutta meridionale il culmine più eccelso della bellezza proprio nel Trovatore». ${ }^{6}$ A questa concezione (che oggi dà fastidio per le sue implicazioni nazionalistiche e irrazionalistiche, ma che allora aveva una

in Verdi's middle period (1849-1859), ed. Martin Chusid, Chicago, University of Chicago Press, 1997, 147-96; M. Chusid, A new source for "El Trovador" and its implications for the tonal organization of "Il trovatore", ivi, 197-225; P. Gallarati, Lettura del "Trovatore", Torino, Libreria Stampatori, 2002; P. Monterde, Sulle canzoni di Azucena e Manrico nel "Trovatore", Studi verdiani 18, 2004, 11-26; W. Osthoff, "Il trovatore". Seine dramatisch-musikalische Einheit und seine tragische Hauptgestalt: Azucena, Studi verdiani 19, 2005, 58-106; A. Gerhard, Giuseppe Verdi, München Beck, 2012, 70-73; M. Chusid, Verdi's "Il trovatore": The quintessential Italian melodrama, Rochester, NY, University of Rochester Press, 2012; G. Paduano, TuttoVerdi. Programma di sala, Torino, EDT, 2013, 89-95 (1 $1^{\mathrm{a}}$ ed. Pisa, ETS, 2001, che sintetizza quanto esposto più ampiamente in Leonora e Azucena: $i$ due universali affettivi, in Il Trovatore, Milano, Edizioni del Teatro alla Scala, 2000, 79-101).

4 Martha Nussbaum, commentando la frase qui riportata in epigrafe, nota: «̇ anche ovvio che certe opere raffinate sono di più facile fruizione di altre .... Ma il fatto che certe opere siano decodificate in modo relativamente facile non implica che non sia necessario alcun apprendistato, e che il codice sia universale» (M.C. Nussbaum, L'intelligenza delle emozioni, Bologna, il Mulino, 2009, 322). Sull'uso che Nussbaum fa della testimonianza di Briggs, si veda L. Bianconi, La forma musicale come scuola dei sentimenti, in Educazione musicale e formazione, a cura di G. La Face Bianconi e F. Frabboni, Milano, Angeli, 2008, 85-120: 114-116 (anche http://www.saggiatoremusicale.it/saggem/ricerca/bibliografia/Bianconi 2008.pdf).

5 Vedi per es. H. Gerigk, Giuseppe Verdi, Potsdam, Athenaion, 1935, 85-87.

6 B. Barilli, Il paese del melodramma (1930), Milano, Adelphi, 2000, 20. 
sua motivazione) si contrappose subito un punto di vista che possiamo definire 'idealistico', rappresentato al meglio, già nel 1933, da Mila:

Proprio il Trovatore [...] non richiede neppure un particolareggiato esame di ogni scena; una sommaria divisione del buono e del cattivo si ha quando si pensi che la vera vita dell'opera non sta nell'intreccio amoroso di Manrico Leonora e il Conte di Luna, ma nel tocco di colore zingaresco che aureola foscamente le figure di Manrico e Azucena. Se si aggiunge a questo elemento la gran scena del Miserere [...] avremo posto da un lato tutto ciò che veramente vive nell'opera: naturalmente non si esclude che anche nel resto non si trovino felici intuizioni musicali, e che questo stesso settore felice languisca a volte in banalità, ma si tratta di particolari che non intaccano l'unità della concezione drammatica, tesa sulla figura di Azucena. ${ }^{7}$

È evidente come questo giudizio presupponesse un tipo ideale di melodramma verdiano esemplato su opere quali Rigoletto, Traviata e soprattutto Otello, in sostanza fondato su un'idea romantico-naturalista del teatro per cui vero dramma è solo quello basato sulla presunta verità psicologica del personaggio. Ma, a parte il fatto che una tale concezione del teatro non è più la nostra, è soddisfacente una lettura che, crocianamente, assegna al regno della poesia un quarto dell'opera, relegando i restanti tre quarti nel dominio di una struttura convenzionale e, si direbbe, fastidiosa?

La centralità di Azucena è comunque restata un punto fermo nella storia della critica successiva. ${ }^{8}$ Essa è confermata anche da un'assai citata osservazione di Verdi:

Parmi [...] che sopratutto Azucena non conservi il suo carattere strano e nuovo: parmi che le due grandi passioni di questa donna Amor figliale, e amor materno non vi siano più in tutta la loro potenza.

Verdi non afferma qui che Azucena sia la chiave di volta dell'intero dramma: si limita a descrivere il conflitto interiore che definisce il per-

7 M. Mila, Il melodramma di Verdi (1933), in Id., L'arte di Verdi, Torino, Einaudi, 1980, 38.

8 Vedi in particolare Osthoff, "Il trovatore", cit.

9 Lettera del 9 aprile 1851, in Carteggio Verdi-Cammarano (1843-1852), a cura di C.M. Mossa, Parma, Istituto Nazionale di Studi Verdiani, 2001 (d'ora in avanti CVC), 190. 
sonaggio. Non è stata altrettanto valorizzata un'altra affermazione, questa volta di Cammarano: «Principalissimo interesse di questo Dramma si è che un fratello uccide l'altro». ${ }^{10}$ Tanto doveva bastare a far sospettare che il sistema drammatico dell'opera sia più complesso di quanto si fosse immaginato, e che l'intreccio amoroso non sia solo un residuo convenzionale che fa da cornice a un moderno dramma psicologico.

Il problema fu radicalmente ripensato da Gabriele Baldini, ${ }^{11}$ a partire dalla cui interpretazione è prevalsa un'idea del Trovatore come capolavoro drammatico, sì, ma esemplare di un tipo di dramma in cui la costellazione dei personaggi va intesa come un sistema di segni e relazioni 'puramente musicali', da indagare quindi con gli strumenti propri dell'analisi musicale e mettendo in secondo piano i dati offerti dal libretto. Che questo orientamento si basasse su un'interpretazione fuorviante del pensiero di Baldini, credo di averlo mostrato altrove in maniera convincente. ${ }^{12}$ Ciò non toglie che gli studi ai quali alludo ${ }^{13}$ abbiano prodotto risultati da tenere nella massima considerazione; resta il fatto che in essi continua a non esser chiaro cosa s'intenda realmente con 'dramma'. D'altronde, che il libretto del Trovatore non possa essere liquidato facilmente basta a dimostrarlo il tempo incredibilmente lungo, senza riscontri per nessuna opera coeva, che fu necessario alla sua gestazione: due anni e mezzo. È dunque da esso che prenderò le mosse, sperabilmente senza ricadere in metodi di lettura superati; considerandolo non come un oggetto meramente letterario o come un dramma compiuto di cui la musica sarebbe l'illustrazione, ma come un testo parziale che è già di per sé musicale, nel senso che Verdi riuscì a farselo confezionare così com'è a partire da un'idea musicale dell'opera che ne sarebbe risultata.

Un vecchio luogo comune sul libretto del Trovatore è la sua oscurità, in particolare il fatto che gran parte della vicenda rimanda a un

10 Lettera del 26 aprile 1851, CVC, 197. Un'eccezione è Osthoff, "Il trovatore", cit., 104.

11 G. Baldini, Abitare la battaglia. La storia di Giuseppe Verdi, a cura di F. d'Amico, Milano, Garzanti, 1970, 234-259.

12 Vedi Della Seta, «...non senza pazzia», cit., 227-38.

13 Fra i titoli elencati alla nota 3, in particolare quelli di Petrobelli, Drabkin, Parker, Chusid, ai quali vanno aggiunti alcuni importanti saggi di Harold S. Powers che non riguardano direttamente Il trovatore. 
antefatto che non viene presentato sulla scena bensì rievocato, e rimane perciò incomprensibile. ${ }^{14}$ Questa accusa fu respinta da Baldini, per il quale esso era anzi

il libretto ideale per musica, e cioè un libretto che consentisse in pieno la vita musicale dei personaggi e quella sola, un libretto, io credo, in sostanza, fantasma, che venisse affatto inghiottito dalla musica e, di per sé, una volta completata l'opera, sparisse. E difatto il libretto del Trovatore è sparito e nessuno l'ha mai più rintracciato. Di nessun'altra opera di Verdi, com'è noto, si può dire come del Trovatore che il libretto non si riesce a raccontare. [...] Ė una qualità tutta speciale di quel testo eletto, e non deriva tanto da complicazioni particolari, quanto dalla natura estremamente elusiva dei personaggi e dell'azione, oltre il segno della musica. [...] Geniale è infatti quel meccanismo del libretto che permette bensì di conoscere la natura della relazione fra il mezzosoprano da una parte e il tenore e il baritono dall'altra, ma non gli accidenti, e che consente a questi di sfuggire sempre a qualsiasi inseguimento. La natura della relazione può, infatti, essere espressa dalla musica, gli accidenti non lo potrebbero. E la natura, com'io credo, è un groviglio di amore e odio, in cui, come sempre accade in Verdi, l'erotismo si mescola sempre agli affetti familiari..$^{15}$

Baldini non contesta, ma dà tutt'altro senso al luogo comune. Ciò detto, bisogna aggiungere che questo, preso alla lettera, non è neppure tanto veritiero. In realtà lo svolgersi dei fatti nella vicenda è chiarissimo

14 Riassume il luogo comune Paduano, TuttoVerdi, cit., 90 sg.: «La mitologia del Trovatore parla di un libretto astruso e sconclusionato, utile a screditare per antonomasia il teatro musicale. In parte questo giudizio è frutto di pigrizie e supponenze; in parte però riflette un'autenticità specifica di quest'opera, esprimibile nella bizzarra formula per cui ciò che accade in essa non è rilevante per i suoi significati: la rappresentazione cioè degli affetti essenziali, l'eros e l'amore materno ....... neanche le antinomie più nette che ne derivano scalfiscono la loro affermazione estrema e categorica. ... la situazione del Trovatore non nasce dal loro conflitto, ma dalla contemporanea presunzione di totalità che ognuno di essi avanza in riferimento alla medesima funzione tenorile, mai apparsa così fragile».

15 Baldini, Abitare la battaglia, cit., 235 e 137. A questa lettura si è opposto energicamente, ancora in epoca recente, e ancora fraintendendo il pensiero di Baldini, Osthoff, "Il trovatore", cit., 59: «Ich setze dem meine These entgegen, dass der Trovatore, deshalb ein grosses Werk der Opernliteratur ist, weil sich ein genialer Komponist an einem ihm adäquate Stoff entzündet und ihm mit Hilfe eines erfahrenen und begabten Textdichters zu einer in sich stimmigen musikalisch-dramatischen Einheit geformt hat». 
da un certo punto in poi, esattamente dalla scena 2 del secondo atto, quando il messo giunge ad annunciare la prossima monacazione di Leonora: da questo momento l'azione si svolge serrata, comprensibile come in qualsiasi altra opera di Verdi. L'oscurità è nella parte precedente, e non dipende dal fatto che gli eventi pregressi siano narrati non è certo l'unico caso - bensì dalla natura stessa di tali eventi. I punti in questione sono sostanzialmente due.

Primo: chi è Manrico? Secondo quanto affermano le ultime parole di Azucena, egli è il figlio del vecchio Conte di Luna e il fratello del giovane, rapito nella culla; la sua morte realizza dunque, sia pure involontariamente, ${ }^{16}$ la vendetta della zingara. D'altra parte, questa considera davvero Manrico suo figlio: lo ha adottato dopo aver bruciato per sbaglio il proprio, e lo ha addirittura 'rigenerato' salvandogli la vita sul campo di battaglia. ${ }^{17}$

Ma le cose stanno proprio così? Non suggerisco che Azucena menta intenzionalmente, tuttavia è opportuno ricordare che, all'interno

16 Involontariamente solo nel libretto dell'opera. Nel dramma spagnolo (qui cit. da A. García Gutiérrez, Il Trovatore, introduzione e traduzione di M. Partesotti, presentazione di P. Menarini, Firenze, Aletheia, 2001) Azucena tenta in extremis, chiedendone perdono allo spirito della madre, d'impedire l'esecuzione di Manrique; quando questa è avvenuta si rivolge a Don Nuño: «iSì, sì... luces... él es: tu hermano, imbécil! ... ¡Ya estás vengada!» (ivi, 207), parole che Verdi, in un appunto per Cammarano, tradusse quasi alla lettera: «Morto Manrico il sentimento della vendetta diviene gigante, e dice con esaltazione... Si Luci luci egli è tuo fratello... Stolto!... Sei vendicata o madre!!» (9 aprile 1851, CVC, 191). Nel suo «programma», scritto all'inizio di aprile 1851, Cammarano aveva eliminato il dilemma di Azucena, salvando solo l'ultima battuta: «Ah! Che facesti?... Egli era... tuo fratello!» (CVC, 187). Il 4 aprile Verdi osservava: «La Gitana non salva sé e Manrique perché sua madre sul rogo le aveva indicato 'Vendicami'» (CVC, 188); il 26 aprile il poeta ribadiva: «La mia Azucena ha il ticchio della vendetta, e non potendo meglio, vuol godere del martirio di De Luna, rammentandogli l'orrenda morte del fratello. ... Un altro tratto mi parve che mancasse nell'originale e ve lo aggiunsi: quello col quale Azucena, anche in mezzo allo scompiglio delle sue idee, rammenta che può salvare Manrique; il pensiero di perderlo col suo silenzio (pensiero d'altronde troppo orribile) abbiamo già veduto non esistere nello spagnuolo, e però credo aver riempiuta una lacuna» (CVC, 197 sg.). Questa affermazione non è, come detto sopra, del tutto vera, ma qui Cammarano si riferisce al primo quadro del terzo atto piuttosto che al finale dell'opera. In conclusione, tutta la questione fu oggetto di attente valutazioni, il che rende tanto più interessante la vaghezza del libretto su questo punto.

17 Vedi Paduano, TuttoVerdi, cit., 94. 
del testo, tutte le informazioni su Manrico ci sono fornite sempre e solo da lei, protagonista e unica testimone del fatto atroce. ${ }^{18}$ Secondo un'ipotesi che, lo ammetto, non è confermata, ma neppure contraddetta, da alcun elemento del testo, ella potrebbe aver ucciso il fanciullo nobile; dopo di che, per un meccanismo di spostamento emotivo alimentato dal rimorso («Ei distruggeasi in pianto... / io mi sentiva il cor dilanïato, infranto!...» ${ }^{19}$ ), potrebbe aver trasferito sul figlio naturale l'identità dell'altro, con tanto successo che il giovane zingaro è cresciuto con un'irresistibile vocazione di cavaliere, capo militare e amante di nobildonne. A un tale processo d'identificazione involontaria si potrebbe attribuire anche il fatto che Manrico abbia risparmiato la vita del Conte, trattenuto da un «moto arcano» e da un grido dal cielo che gli disse «non ferir!». Dunque, dal suo punto di vista, Azucena direbbe il vero sia quando rassicura Manrico che egli è suo figlio sia quando grida al Conte: «Egli era tuo fratello!». Piuttosto, è strana la domanda di Manrico «Non son tuo figlio?... E chi son io? chi dunque?...». Se egli rifiuta di prendere in considerazione la possibilità di essere il bambino salvato, la domanda giusta sarebbe: «Ma allora, che fine fece il figlio del Conte?». Non si vede infatti perché Azucena non potrebbe aver avuto un altro figlio 'vero'. ${ }^{20}$

Secondo: nel corso dell'opera Manrico e il Conte si scontrano tre volte, alla fine di ciascuno dei primi tre atti. Ma quando e come si sono scontrati in passato? Nel dramma spagnolo la successione è chiara. I

18 Questa precisazione è essenziale per tutto il ragionamento che segue. Quando parlo di 'verità', intendo sempre quella che vale all' interno del testo, l'unica che conta in un discorso di finzione: relativamente al mondo immaginario in cui vivono $\mathrm{i}$ personaggi, non ha nessuna rilevanza ciò che gli autori eventualmente ritenevano circa la loro 'realtà' (vedi oltre la nota 20), che solo in alcuni casi può essere fortuitamente documentato e che il destinatario non è comunque tenuto a sapere.

19 Il testo del Trovatore è citato da Libretti d'opera italiani dal Seicento al Novecento, a cura di G. Gronda e P. Fabbri, Milano, Mondadori, 1997, 1365-1404. Per il testo musicale, si veda l'edizione critica: G. Verdi, Il trovatore, ed. D. Lawton, Chicago-Milano, University of Chicago Press-Ricordi, 1992 (The Works of / Le opere di Giuseppe Verdi, s. 1, vol. 18A); grazie a questo strumento è possibile verificare le numerose varianti introdotte dal compositore nel testo poetico, che menzionerò quando significative nel contesto di questo saggio.

20 «È importante che Manrico non sappia se è veramente suo figlio, è importante che Azucena si smentisca continuamente attorno a questo problema, è importante che ella si senta come proiezione di un'altra madre, di un'altra zingara che s'è trovata in 
duelli individuali sono due, il primo dei quali, rievocato dai famigli (I, 1), è avvenuto qualche tempo prima dell'inizio dell'azione: «Il combattimento fu breve; la spada del Conte cadde ai piedi del suo rivale e un attimo dopo in giardino non c'era più anima viva $\gg^{21}$ (non v'è cenno di strane pietà, di moti arcani e di voci dal cielo). Al secondo duello assistiamo $(I, 5)$ fino al momento in cui i due si allontanano con le spade sguainate, come nel primo atto dell'opera; successivamente (II, 1) apprendiamo, sempre dai famigli, che in esso Don Nuño fu ferito. Nel frattempo è passato un anno, durante il quale ha avuto luogo la battaglia di Velilla (rievocata in II, 1, II, 4 e II, 7), dove Don Manrique fu creduto morto; ma in realtà egli non era presente sul campo di battaglia, trovandosi a quell'epoca in Castiglia, e dunque non fu mai ferito né salvato da Azucena.

Il ferimento di Manrico - così come la sua «strana pietà» - fu introdotto da Cammarano e Verdi. Esso diede occasione a una discussione tra i due se dovesse essere accaduto nel duello (ridotto a uno solo) o in battaglia. ${ }^{22}$ Verdi ebbe la meglio, ma, come il poeta aveva ben

una situazione analoga cui ora essa deve porre il suggello della vendetta, ma è importante soprattutto che chi ascolta questa musica di una chiarezza abbagliante sovrapponga continuamente questi personaggi l'uno sull'altro e non ne sappia mai districare le sagome» (Baldini, Abitare la battaglia, cit., 251 sg.). In realtà un indizio che Azucena sia consapevole di mentire c'è: la didascalia secondo cui ella risponde a Manrico: «Tu sei mio figlio!» «con la sollecitudine di chi cerca emendare involontario fallo». Ma, a parte il fatto che anche questo indizio testuale può essere collocato nel gioco tra verità e finzione che caratterizza il personaggio (vedi sopra), esso mi sembra piuttosto rivelatore di una preoccupazione del librettista. Il 4 aprile 1851 Verdi osservava: «È vero che la Gitana fa intendere che Manrique non è suo figlio, ma è una parola che le sfugge nel racconto e che la ritira sì presto che il Trovatore, lontano dal pensare cosa simile, non può credere sia quella una verità» $(C V C, 188)$; fatto sta che in partitura egli sostituì la didascalia del libretto con un elusivo «interrompendo», così come corresse il lapsus rivelatore del libretto, «col figlio... teco in braccio», nel più neutro «col figlio... sulle braccia». Si veda anche la puntigliosa disamina di questo aspetto nella lettera di Cammarano del 26 aprile 1851 (CVC, 197, in particolare: «Quando Azucena non ragiona, ragiona meglio il Dramma»). Invece nel testo spagnolo manca qualsiasi ambiguità e il dialogo è condotto all'insegna della logica: «MANRIQUE: Vostro figlio?! Ma allora, chi sono io, chi? Tutto è chiaro. AzuCENA: Ti ho detto di aver bruciato mio figlio? No... Ho voluto prendermi gioco della tua ambizione: tu sei mio figlio!» (García Gutiérrez, Il Trovatore, cit., 123-5).

21 García Gutiérrez, Il Trovatore, cit., 75.

22 Vedi CVC, 185 (programma di Cammarano: racconto del ferimento in duello); 190 (Verdi: «non amerei che il Trovatore restasse ferito nel Duello»; «Trovatore ferito in battaglia»). 
visto, ${ }^{23}$ il risultato non è facilmente afferrabile nel testo e soprattutto nella rappresentazione scenica. Al calar di sipario del primo atto vediamo Manrico e il Conte in procinto di battersi; all'inizio del secondo troviamo Manrico ferito e curato dalla madre. Data la stretta concatenazione, è logico pensare che il ferimento sia avvenuto proprio nel duello. Più avanti apprendiamo che Leonora fu «tratta in inganno di [sua] morte al grido»; poiché essa era fisicamente vicina al luogo del duello, siamo indotti a credere che ella abbia realmente udito il grido di Manrico nel momento in cui cadeva. Ma una lettura attenta di uno dei luoghi letterariamente più aulici del libretto, per di più enunciato in un recitativo da dirsi «molto presto», c'informa che Manrico fu invece ferito «nei pugnati campi / di Pelilla, ove spento fama [lo] disse», e dove Azucena mosse «notturna» a dargli sepoltura. Il «grido» che raggiunse Leonora era dunque, figuratamente, la fama. Invece «nel singolar certame», avvenuto qualche tempo prima della battaglia campale, Manrico aveva avuto la meglio, pur risparmiando la vita del Conte. Sulla carta i conti tornano, ma qualcosa di poco chiaro c'è. Ancora: perché alla fine del secondo atto Manrico, avendo in suo potere il rivale, non solo gli risparmia ancora la vita - contravvenendo al giuramento fatto poco prima alla madre - ma neppure lo prende prigioniero, come gli imporrebbe il suo dovere di capo militare ${ }^{24}$ (Di nuovo, il problema riguarda solo l'opera, dato che nel dramma spagnolo il rapimento di Leonor si svolge in modo molto diverso).

Tutto ciò sembra essere di scarsa utilità per la comprensione della vicenda, ma sappiamo che la struttura temporale è un aspetto importante per la determinazione del senso di un'opera drammatica e, piuttosto che liquidare come assurda la vicenda del Trovatore, conviene assumerne l'assurdità' come un dato da interpretare. Non che essa sia stata intenzionalmente ricercata dagli autori: è piuttosto il risultato di una mediazione tra visioni diverse, cui si aggiunsero, come al solito, la

23 Ibid., 195: «Manrique ferito in duello annoda meglio la seconda Parte alla prima, e non astringe a cercare altre cagioni ed altri schiarimenti per motivare la supposta morte di lui».

24 Un risposta la forniva una prima stesura del libretto, in cui il Finale del secondo atto prevedeva una 'stretta' coi seguenti versi di Manrico: «Sia respinto quest'uomo insensato... / Morte invano egli spera da me... / Vivi, e renda il sapermi beato / Un supplizio la vita per te» $(C V C, 219)$. 
difficoltà di condensare la complessa vicenda originale e la necessità di aggirare le prevedibili obiezioni della censura. Ma tutto ciò appartiene alla storia genetica del testo; una volta compiuto quest'ultimo, l'oscurità è divenuta una caratteristica strutturale dell'opera e come tale dobbiamo affrontarla.

$\mathrm{Ci}$ viene in aiuto ancora una volta Baldini, non nelle pagine sul Trovatore ma nell'autocommento al proprio 'dramma' postumo Selva e torrente, che egli dichiara essere una realizzazione del proprio sogno giovanile di «scrivere delle opere liriche nello stile di Bellini Verdi Wagner Strauss»:

La serie temporale, come viene avvertito più volte nel corso del dramma, non corrisponde a una successione cronologica convenzionale. Le scene evocate si susseguono secondo criteri diversi da quelli cronologici. Tutto quel che non si capisce e tutti i conti che non tornano sono tali per decisa volontà dell'autore; non già per bizzarria, ma perché è sembrato veramente che la cosa non avesse importanza alcuna in siffatto genere di composizione. $^{25}$

Letta in questa prospettiva, l'oscura struttura drammatica del Trovatore, lungi dall'essere il frutto di un compromesso, sembra evitare intenzionalmente la linearità narrativa del dramma 'classico', per cui gli eventi si succedono irreversibilmente secondo una connessione causale, in favore di una specie di spazio-tempo nel quale ogni evento si colloca prima e dopo ogni altro e può quindi essere ripercorso all'infinito. ${ }^{26}$ Invece di cercare di stabilire la successione esatta degli scontri tra Manrico e il Conte di Luna, potremmo dire che essi si sono scontrati e si scontreranno ancora - un numero indeterminato di volte. Questo tipo di struttura temporale ciclica è caratteristico del racconto di tradizione orale nelle sue diverse manifestazioni: del mito, della leggenda,

25 G. Baldini, Selva e torrente, Torino, Einaudi, 1970, 138, cit. e commentato in Della Seta, «...non senza pazzia», cit., 238.

26 Vedi per es. P. Gallarati, Il melodramma ri-creato. Verdi e la 'trilogia popolare', in: Finché non splende in ciel notturna face. Studi in memoria di Francesco Degrada, a cura di C. Fertonani et al., Milano, LED, 2009, 171-85, in particolare 174: «il tempo nel Trovatore ... non è quello empirico dei fenomeni, ma quello metafisico dell'immaginazione. Qui non contano tanto i fatti ma le visioni che, sorprendentemente, si avverano, cancellando la differenza tra passato, presente e futuro». 
della fiaba. ${ }^{27}$ Possiamo allora leggere la peculiarità del Trovatore nel fatto che si tratta della riproposizione in chiave operistica di un tema tradizionale, ciò che qualche volta è stato espresso notando in esso aspetti di leggenda o di favola tragica. ${ }^{28}$

Motivi fiabeschi non mancano nel Trovatore, per esempio quello del figlio sottratto dalla culla e creduto morto: nel Novecento Pirandello ne fece una novella (Le nonne, 1902, rifatta come Il figlio cambiato, 1925) e poi un libretto d'opera per Gianfrancesco Malipiero, La favola del figlio cambiato (1932-33); nel frattempo aveva pensato d'inglobare la favola nel 'mito' I giganti della montagna. ${ }^{29}$ E dopo tutto anche l'Orco di Pollicino uccide per sbaglio le proprie figlie invece dell'eroe e dei suoi fratellini.

Un tema di cui sono pieni i miti, le leggende e le letterature di tutti i tempi e di tutti i popoli è quello dei 'fratelli nemici'.$^{30}$ Fra le innumerevoli varianti di esso, una c'interessa particolarmente: quella secondo cui i fratelli si scontrano e si uccidono - o uno uccide l'altro - per amore

27 Il rapporto d'identità ovvero diversità tra queste diverse forme di narrazione è stato ed è oggetto di dibattiti assai vivi. Si veda per tutte la discussione tra LéviStrauss e Propp in V. J. Propp, Morfologia della fiaba, con un intervento di C. LéviStrauss e una replica dell'autore, Torino, Einaudi, 1966, 96, 80 sg., 223 sg. Sulla struttura temporale del mito, vedi Lévi-Strauss, ivi, 192: in ogni sistema mitico «la narrazione è insieme "nel tempo" (consiste in una successione di eventi) e "fuori dal tempo" (il suo valore significante è sempre attuale)».

28 Sulla nozione diffusa del Trovatore come narrazione epico-leggendaria si basa la reinterpretazione offertane da Luciano Berio in La vera storia (1982): in una lettera a Vittorio Sermonti del 10 gennaio 1975 Berio definisce «tutto quello che è successo nella prima parte» della sua futura opera «la rappresentazione di una lunga e complessa ballata sulla vicenda del "Trovatore"», mentre «la II parte sarà una ballata scenica di una ballata di una ballata...» (pubblicata in M. Girardi, "Il trovatore" nel 1982 secondo Berio, Calvino e Sermonti in "La vera storia", in Verdi 2001. Atti del Convegno internazionale ... 24 gennaio - $1^{\circ}$ febbraio 2001, a cura di F. Della Seta et al., Firenze, Olschki, 2003, 458). Si veda in proposito L. Zoppelli, Dramaturgie structurale? Nouvelles observations sur le rapport entre "La vera storia" et "Il trovatore", relazione tenuta nella terza giornata del ciclo "Dramaturgie musicale contemporaine en Europe - Le théâtre musical de Luciano Berio”, Parigi, Université de Paris 8, 18 novembre 2011 (si può leggere a http://www2.univ-paris8.fr/DMCE/page.php?page=9\#LVS).

29 Vedi Luigi Pirandello, Maschere nude, a cura di A. d'Amico con la collaborazione di A. Tinterri, vol. 4, Milano, Mondadori, 2007, 721.

30 L'idea che «i fratelli hanno il medesimo significato dei nemici» apparteneva 
della stessa donna ${ }^{31}$ (parlando di opera viene subito in mente Pélleas et Mélisande, una leggenda drammatica fuori del tempo). Ne troviamo una versione antichissima già nel Mahābhārata: i fratelli Sunda e Upasunda si uccidono a vicenda per amore della bellissima Tilottāma, inviata dagli dei per punire la loro arroganza. ${ }^{32}$ Difficilmente Verdi e

alla saggezza popolare dell'Antichità: «Essi infatti non tornano a vantaggio bensì a danno, proprio come i nemici: perché ciò che uno avrebbe avuto da solo, lo deve dividere a metà oppure in tre parti con i fratelli» (Artemidoro, Il libro dei sogni, IV, 70, a cura di D. Del Corno, Milano, Adelphi, 1975, 258). Un gran numero di variazioni su questo tema nella letteratura occidentale, da Esiodo a Steinbeck (East of Eden), è illustrato in E. Frenzel, Motive der Weltliteratur. Ein Lexikon dichtungsgeschichtlicher Längsschnitte, Stuttgart, Kröner, 1976, 80-94. In ambito psicoanalitico sono disponibili due studi recenti: L. Kancyper, Il complesso fraterno. Studio psicoanalitico, Roma, Borla, 2008 (ed. or.: El complejo fraterno. Estudio psicoanalitico, Buenos Aires, Lumen, 2004); R. Kaës, Il complesso fraterno, Roma, Borla, 2009 (ed. or.: Le complexe fraternel, Paris, Dunod, 2008).

31 Osthoff, "Il trovatore", cit., 104, fa riferimento al tema, con richiami a Eschilo, Schiller e Byron, ma non dà evidenza alla sua variante erotica. Peraltro il conflitto tra fratelli (ovvero tra sorelle) era già stato trattato da Verdi in Nabucco, I Lombardi/Jérusalem, I masnadieri, e da Cammarano per Donizetti in Maria de Rudenz (1838). Inoltre esso è centrale in Shakespeare (vedi J. T. McCullen, Brother hate and fratricide in Shakespeare, Shakespeare Quarterly 3, 1952, 335-40): è significativo che nel progetto di Re Lear abbozzato da Verdi e Cammarano nel 1850 (CVC, 166-9 e 366-78) al tema fosse dato un rilievo notevole, mentre nelle varie stesure del libretto elaborate con Antonio Somma tra il 1853 e il 1856 (vedi Carteggio Verdi-Somma, a cura di S. Ricciardi, Parma, Istituto nazionale di studi verdiani, 2003, 41-182) esso venne progressivamente messo da parte, quasi che Verdi ritenesse di averne esaurito le potenzialità drammatiche nelle opere precedenti.

32 Ha richiamato la mia attenzione su questo racconto un meraviglioso bassorilievo cambogiano del X secolo esposto al Musée Guimet di Parigi (dal frontone del tempio di Banteay Srei; visibile a http://www.flickr.com/photos/dalbera/6364409273). L'episodio è narrato nel primo libro del poema, che si può leggere in inglese in The Mahābhārata, 1, The Book of Beginning, translated and edited by J.A.B. van Buitenen, Chicago-London, The University of Chicago Press, 1983. Ne cito qui la conclusione (ivi, p. 397 sg.): «Maddened by the boon they had received, by the strength of their chests, by their riches and gems, by the liquor they had drunk, maddened by all these madnesses, they knitted their brows at each other and, possessed by crazed lust, began to speak to each other. "She is my wife", said Sunda, "and your guru!" "She is mine", quoth Upasunda, "and your sister in law!" Rage seized them: "She is not your, she is mine!" And for her they grasped their horrible clubs. When they had grasped their horrible clubs, they hit each other shouting "Me first, me first!" - blinded by their love for 
Cammarano (nonché García Gutiérrez) avranno conosciuto questa storia, ma sicuramente conoscevano La sposa di Messina (1803) di Schiller, di cui Andrea Maffei aveva pubblicato una traduzione. ${ }^{33}$ E non è impossibile, sebbene improbabile, che l'uno o l'altro conoscesse la ballata Zwei Brüder (1820) di Heinrich Heine,$^{34}$ messa in musica da Schumann nel 1840 col titolo Die feindlichen Brüder. ${ }^{35}$ Ispirata a un'antica leggenda renana ${ }^{36}$ narra di due fratelli che, per amore della Contessa Laura, «si battono in furioso duello, accesi d'odio» fino a cadere «l'uno sulla spada dell'altro». Così si conclude il racconto:

Viel Jahrhunderte verwehen,

Viel Geschlechter deckt das Grab;

Traurig von des Berges Höhen

Schaut das öde Schloß herab.

Aber nachts, im Talesgrunde,

Wandelt' s heimlich, wunderbar;

Wenn da kommt die zwölfte Stunde,

Kämpfet dort das Brüderpaar.
Passano molti secoli, molte generazioni copre la tomba; triste dall'alto della montagna guarda giù il castello deserto.

Ma di notte, nel fondo della valle, sinistri portenti si aggirano; al suonar della mezzanotte, là combattono i due fratelli. ${ }^{37}$

Compare qui lo schema ciclico, che si presta a essere narrato e rinarrato. E stato notato ${ }^{38}$ che la scena iniziale del Trovatore, in cui i famigli chiedono a Ferrando di raccontare «la vera storia di Garzia, ger-

her. Clobbered by the clubs, the terrible pair fell to the ground, their bodies smeared with blood, like two suns falling from heaven».

33 La sposa di Messina, tragedia di F. Schiller, traduzione del cavaliere Andrea Maffei, Milano, Fontana, 1827. Un'altra traduzione, dell'avvocato Antonio Caimi, fu pubblicata sempre a Milano da Sonzogno nel 1828.

34 Nella raccolta Romanzen, del 1821, pubblicata nel Buch der Lieder del 1827. La poesia non è compresa tra quelle tradotte da Gérard de Nerval nella Revue des deux mondes, 15 luglio 1848, 224-43, e 15 settembre 1848, 914-30 (e neppure nella successiva serie apparsa ivi, 1 luglio 1854, 354-76); né risulta che fosse stata tradotta in italiano.

35 È il n. 2 delle Romanzen und Balladen op. 49, pubblicate nel 1844.

36 H. Jacke, Die rheinische Sage von den feindlichen Brüdern in ibrer von der Romantik beeinflußten Wirkung, Wuppertal-Elberfeld, Martini \& Grüttefien,1932 (Beiträge zur rheinischen und westfälischen Volkskunde in Einzeldarstellungen, Heft 7).

37 Cit. da H. Heine, Sämtliche Gedichte in zeitlicher Folge, Frankfurt a.M., Insel 2007, 79-80; mia la traduzione, per la quale sono debitore ai consigli di Andrea Landolfi.

38 Vedi M. Mila, La giovinezza di Verdi, Torino, ERI, 1974, 451. 
mano al nostro Conte», anticipa quella del Parsifal (1882): anche lì gli scudieri chiedono al vecchio Gurnemanz di raccontare «la vera storia» della ferita del Re: «Però, piccolo padre, di' e insegnaci a punto: / tu conoscesti Klingsor: - com'è avvenuto?»; e all'inizio del Crepuscolo degli dei le Norne si raccontano, come hanno fatto infinite volte, la storia che anche noi abbiamo già visto: «canta, o sorella, - io a te la lancio - sai tu, questo come avvenne?». ${ }^{39}$

Non ho fatto a caso il nome di Wagner. È infatti significativo che esattamente negli stessi anni, senza saper nulla l'uno dell'altro, i due massimi drammaturghi musicali dell'Ottocento abbiano deciso di ricorrere al mito, l'uno sotto le vesti della favola tragica, l'altro nella sua forma più pura: la concezione dell'Anello del Nibelungo coincide con quella del Trovatore, ed entrambi ebbero l'idea di assumere il fuoco come tema 'musicale'40 (non c'è bisogno di ricordare le valenze simboliche che questo tema ha nella tradizione mitica universale e nell'immaginario della cultura europea).

Com'è noto, per Wagner il ricorso al mito ha un valore ideologico: solo grazie ad esso è possibile ritrovare il fondo 'puramente umano' dei sentimenti degradato dal convenzionalismo dei soggetti storici dell'opera italiana e francese. Verdi era di opinione diversa: tutta la sua opera è volta ad esplorare la complessità dell'animo umano, delle sue passioni, delle relazioni interumane, sempre collocate in un preciso, seppur idealizzato, contesto storico e culturale. ${ }^{41} \mathrm{Ma}$ è azzardato supporre che, almeno per una volta, egli sia stato tentato dall'idea di rappresentare le grandi passioni in sé e per sé, nella loro forza brutale e irrazionale, e sia per questo rimasto affascinato da un soggetto così lontano dalla linea maestra della sua drammaturgia, soprattutto nella forma in cui egli lo forgiò a partire dal suo modello letterario? Questo

39 Rispettivamente: Parsifal, I, vv. 159-60; Götterdämmerung, Vorspiel, vv. 413, nelle traduzioni di Guido Manacorda, Firenze, Sansoni, 1982 e 1974.

40 Vedi Baldini, Abitare la battaglia, cit., 257-9.

41 Di tal genere era anche la vicenda del Trovador di García Gutiérrez, della quale è precisata persino l'esatta cornice temporale: dal 1390 al 1412. Contrariamente alle sue abitudini, Verdi eliminò - o lasciò che Cammarano eliminasse - tutti i riferimenti storici essenziali, anche se nel libretto rimase, chi sa perché, una data precisa, il 1409. Il palazzo dell'Aljafería di Saragozza, la cui principale attrazione turistica è la Torre del Trovador, ha assunto contorni leggendari proprio grazie alla popolarità di cui gode il dramma in Spagna. 
spiega anche l'apparente illogicità del libretto: essa è tale rispetto alle leggi della logica aristotelica, ma, come ci ha insegnato un secolo di psicoanalisi, la vita emotiva ne segue una propria totalmente diversa, che si ripercuote sulle nostre azioni coscienti con risultati imprevedibili. ${ }^{42}$

Non è senza qualche resistenza che m'induco a ricorrere, per completare il mio ragionamento, a concetti e termini attinti al pensiero di Sigmund Freud. Mi sono convinto a farlo con due precisazioni. In primo luogo, qualunque cosa si pensi della solidità scientifica della psicoanalisi, del suo impianto teorico e della sua validità terapeutica, credo che nessuno possa mettere in dubbio l'importanza di Freud come espressione e autocoscienza di aspetti fondamentali della civiltà occidentale, in particolare della cultura ottocentesca. Non è necessario condividere la sua teoria del complesso di Edipo o quella del narcisismo per riconoscere la straordinaria importanza che i miti da cui esse prendono nome hanno avuto nella formazione e nello svolgimento della coscienza europea, ${ }^{43}$ e quindi utilizzare le interpretazioni freudiane come reagenti utili a ritrovarne le tracce nei testi che analizziamo.

In secondo luogo, uso il pensiero freudiano nell'unico modo oggi possibile nell'ambito della critica d'arte, quello che è ci è stato magistralmente insegnato da Francesco Orlando nelle sue analisi letterarie e nei suoi scritti di teoria della letteratura: ${ }^{44}$ non banale psicologismo del-

42 Che l'inconscio segua nelle sue manifestazioni una logica diversa da quella del pensiero cosciente è idea presente già nella Interpretazione dei sogni; se ne veda la formulazione sintetica in S. Freud, Introduzione alla psicoanalisi, in Opere complete, versione digitale, Torino, Bollati Boringhieri, 2013, vol. 8, 5566. Tale idea ha trovato il suo sviluppo più sistematico nell'opera di Ignacio Matte Blanco, che, per spiegare il funzionamento della vita emotiva, ha postulato una 'bi-logica', e successivamente una 'bimodalità' in cui coesistono due modi di pensiero, asimmetrico e simmetrico (si vedano i suoi L'inconscio come insiemi infiniti. Saggio sulla bi-logica, Torino, Einaudi, 1981; e Pensare, sentire, essere. Riflessioni cliniche sull'antinomia fondamentale dell'nomo e del mondo, Torino, Einaudi, 1995).

43 Vedi G. Paduano, Lunga storia di Edipo Re. Freud, Sofocle e il teatro occidentale, Torino, Einaudi, 1994 (nuova ed.: Edipo, storia di un mito, Roma, Carocci, 2009); M. Bettini, E. Pelizer, Il mito di Narciso. Immagini e racconti dalla Grecia a oggi, Torino, Einaudi, 2003.

44 Si vedano, di Orlando, i volumi poi riuniti nella trilogia dal titolo complessivo Letteratura, ragione e represso. Tre studi freudiani: Due letture freudiane: Fedra e Il misantropo, nuova ed. ampliata, Torino, Einaudi, 1990; Per una teoria freudiana della 
l'autore o del personaggio, ma concezione del testo letterario - nel nostro caso drammatico-musicale - come sistema simbolico strutturato secondo principi formali analoghi a quelli che governano le manifestazioni dell'inconscio. Questa prospettiva ci consente d'inquadrare da un punto di vista solo apparentemente diverso due nodi che ho già spiegato facendo appello alle strutture del racconto mitico. ${ }^{45}$ Se Manrico sia davvero il figlio della zingara o invece del vecchio Conte è un problema per la logica diurna, non per quella dell'inconscio, che non conosce il principio di non contraddizione, come pure può ignorarlo il racconto: all'interno di questo - diciamo nella prospettiva di Azucena - Manrico è realmente l'uno $e$ l'altro. ${ }^{46}$ Non è un problema neppure quante volte e in che ordine abbiano combattuto i fratelli rivali, dato che l'inconscio

letteratura, nuova ed. ampliata, ivi 1987; Illuminismo, barocco e retorica freudiana, nuova ed. ampliata, Torino, Einaudi, 1997; e inoltre il suo Saggio introduttivo a S. Freud, Il motto di spirito e la sua relazione con l'inconscio, Torino, Boringhieri, 1975, 15 29. Il pensiero di Orlando, elaborato all'inizio degli anni Settanta del Novecento, fu influenzato in un primo tempo dalla lettura di Lacan, poi in maniera decisiva da quella di Matte Blanco; vedi in proposito S. Timpanaro, F. Orlando, Carteggio su Freud (1971-1977), Pisa, Scuola normale superiore, 2001. Per una messa a punto aggiornata della problematica si veda G. Paduano, Il testo e il mondo. Elementi di teoria della letteratura, Torino, Bollati Boringhieri, 2013, 110-23.

45 Per dirla con le parole di un grande antichista, che peraltro ha criticato l'interpretazione freudiana di Edipo: «Il mito non si definisce solo attraverso la sua polisemia, l'incastro dei diversi codici gli uni negli altri. Fra i termini che distingue o che oppone nella sua armatura categoriale, esso organizza, nello svolgimento narrativo e nella divisione dei campi semantici, passaggi, divisioni, slittamenti, tensioni, oscillazioni, come se i termini pur escludendosi, s'implicassero anche in certo qual modo. Il mito mette dunque in gioco un tipo di logica che si può chiamare, per contrasto con la logica di non contraddizione dei filosofi, una logica dell'ambiguo, dell'equivoco, della polarità. Come formulare o formalizzare queste operazioni di senso alterno che rovesciano un termine nel suo contrario pur tenendoli per altri aspetti a distanza? Tocca al mitologo prendere atto, in conclusione, di questa carenza, rivolgendosi ai linguisti, ai logici, ai matematici perché gli forniscano lo strumento che manca: il modello strutturale di una logica che non sia quella binaria del sì o no, di una logica diversa dalla logica del lógos» (J.-P. Vernant, Mito e società nell'antica Grecia, seguito da Religione greca, religioni antiche, Torino, Einaudi, 1981, 250). Il modello invocato da Vernant si trova appunto nell'opera di Matte Blanco, apparsa nello stesso anno, il 1975, in cui egli scrive.

46 Nei termini di Matte Blanco (vedi sopra, nota 42), secondo cui il pensiero simmetrico delle emozioni (che per manifestarsi deve necessariamente appoggiarsi al pensiero asimmetrico che governa il linguaggio cosciente) identifica ogni parte di un 
fa, e il racconto può fare a meno di successione temporale lineare, prima e dopo, rapporti irreversibili di causa ed effetto.

Non sono certo il primo a ricorrere, o almeno ad alludere, a concetti freudiani a proposito del Trovatore. ${ }^{47}$ Molti anni fa Pierluigi Petrobelli - notando la similarità di due frasi melodiche di Leonora e di Azucena, entrambe espressione del loro amore per Manrico - osservava che «solo le teorie dei moti più profondi dell'inconscio possono spiegare un parallelismo tanto straordinario».$^{48}$ Io stesso ho fatto allusione alla teoria freudiana dell'atto mancato quando, confrontando la scena finale del Trovatore con quella de La Juive di Scribe e Halévy, osservavo che «la situazione è molto simile ... salvo che qui la scelta che risolve il conflitto non è frutto di deliberato fanatismo ma, con maggiore sottigliezza psicologica, di un'omissione: in Azucena il desiderio di vendetta ha la meglio facendole 'dimenticare' di rivelare in tempo la vera identità di Manrico».49 Anche Anselm Gerhard ha confrontato Il trovatore con La Juive, individuando nel primo il superamento del concetto di 'fatalità' tipico del teatro romantico francese degli anni Trenta:

Azucena [...] spinta dalla sua ossessione, è un personaggio attivissimo, il suo destino non le dà tregua prima del compimento di un atto distruttivo che riproduce l'evento traumatico dell'antefatto. [...] E anche chi trovi anacronistica la conclusione che la favola del Trovatore anticipa la teoria freudiana del Wiederholungszwang, della 'coazione a ripetere', potrà essere d'accordo

insieme con la totalità di questo: il figlio è tutti i figli, la madre è tutte le madri. Dato che in questo regime logico non vige il principio di non contraddizione (A è anche nonA), le due ipotesi circa l'identità di Manrico (vedi sopra, nota 20) non sono mutuamente esclusive: il grido di Azucena «Mio figlio avea bruciato!» e la sua affermazione «Tu sei mio figlio!» sono entrambi 'veri' alla luce della doppia e complementare inferenza $a$ ) «Ho bruciato mio figlio, dunque tu, il figlio del Conte, sei lui»; $b$ ) «Ho bruciato il figlio del Conte, dunque tu, mio figlio, sei lui». Quanto ciò sia 'vero' lo può verificare su sé stesso chiunque, soprattutto se genitore, provi a immaginare di uccidere in quel modo un bambino.

47 Rispetto a Rigoletto e La traviata, «il Trovatore è, come tutti sanno, una faccenda più oscura e freudiana» (L. Berio, Verdi?, Studi verdiani 1, 1982, 99-105, cfr. 102).

48 Petrobelli, La musica nel teatro, cit., 117. Questa osservazione sembra una conferma anticipata, basata sul testo musicale, della chiave interpretativa di Paduano cit. alla nota 14 .

49 F. Della Seta, Italia e Francia nell'Ottocento, Torino, EDT, $1995^{2}$ (Storia della musica, a cura della Società italiana di musicologia, vol. 9), 232. 
almeno sul fatto che Verdi ha saputo darci un'immagine delle passioni che spiega l'odio cieco meglio del ricorso ai poteri sovrumani del fato. ${ }^{50}$

La coazione a ripetere - a rivivere e riprodurre situazioni traumatiche - è alla base di una delle più affascinanti e controverse idee freudiane, quella che vede la vita psichica come un intreccio inestricabile tra pulsione di vita, finalizzata alla riproduzione dell'individuo e alla conservazione della specie, e pulsione di morte, che lavora per ricondurre l'organismo all'inerzia della materia inorganica. ${ }^{51}$ Ancora una volta, quale che sia lo stato del dibattito scientifico su questa teoria, ${ }^{52}$ essa è dichiaratamente ispirata a concezioni primordiali - rappresentate in termini mitologici dall'opposizione tra Eros e Thanatos - che

50 Gerhard, Dalla fatalità all'ossessione, cit., 66. In una linea interpretativa simile si veda ora anche E. d'Angelo, Il trovatore, in Leggendo libretti. Da "Lucia di Lammermoor" a "Turandot", Roma, Aracne, 2013, pp. 77-101.

51 La prima e fondamentale esposizione di tale idea si trova nel saggio del 1920 Jenseits des Lustprinzips (Al di là del principio di piacere, in Freud, Opere complete, cit., vol. 9, 4376-4475), che avviò una vera svolta del pensiero freudiano. Essa circola negli scritti successivi dell'autore senza essere più ripresa organicamente. Si veda l'esposizione sintetica in Id., Introduzione alla psicoanalisi, ivi, vol. 8, 5589-5594.

52 Ho fatto riferimento a strumenti d'informazione affidabili quali J. Laplanche, J.Bertrand Pontalis, Enciclopedia della psicoanalisi, nuova edizione a cura di L. Mecacci e C. Puca, Roma-Bari, Laterza, 20109, in particolare 483-491, e S. Vegetti Finzi, Storia della psicoanalisi. Autori opere teorie 1895-1990, Milano, Mondadori, 2011, in particolare 117-9. Come osserva Vegetti Finzi: «La teoria delle pulsioni di morte rappresenta il momento più alto della speculazione freudiana. ... essa contribuirà ben poco alle successive elaborazioni teoriche. ... Sarà Melanie Klein a riprendere vigorosamente, nel cuore del suo apparato teorico, questo tragico dualismo destinato a rimanere, nel campo freudiano, un fondamentale patrimonio di saggezza. Per Freud, infatti, il principio di morte è un'istanza che si colloca al di là dell'esperienza psicologica; è un postulato necessario per spiegare una serie di fenomeni che rimarrebbero altrimenti inintelligibili. Come tale reintroduce la metafisica proprio in quella teoria psicologica che si era proposta, ai suoi esordi, di scacciare coi suoi lumi le tenebre del sopra-sensibile» (ivi, 119). Fra i continuatori di Freud, il tema è fondamentale, oltre che nel pensiero di Klein, in Lacan (si veda ora in proposito M. Recalcati, Jacques Lacan. Desiderio, godimento e soggettivazione, Milano, Cortina, 2012, 281-90). La più energica contestazione dell'interpretazione freudiana è quella di E. Fromm, Anatomia della distruttività umana, Milano, Mondadori, 1975. Il problema è poi centrale nel più ampio dibattito filosofico sul male e la distruttività, su cui si veda ora S. Forti, I nuovi demoni. Ripensare oggi male e potere, Milano, Feltrinelli, 2012, da leggere tutto ma in particolare, su Nietzsche e Freud, le pp. 52-89 (ringrazio Michela Garda per avermi segnalato questo interessante studio e per le molte stimolanti conversazioni su questi temi). 
nell'Ottocento trovarono espressione nella filosofia di Arthur Schopenhauer ${ }^{53}$ e, per adesione e poi per reazione, in quella di Friedrich Nietzsche: due nomi fondamentali ${ }^{54}$ della costellazione intellettuale comunemente evocata per interpretare il pensiero e l'opera di Wagner, la cui produzione matura, dal Ring al Parsifal, è interamente dominata da questa problematica. Ė troppo azzardato invocare la stessa costellazione per aiutarci a capire meglio un'opera del suo grande contemporaneo? Un'opera che, come abbiamo visto, è più vicina di quanto si pensasse un tempo alle ragioni profonde dell'arte wagneriana? ${ }^{55}$

La mia tesi è dunque che nel Trovatore Verdi abbia dato espressione al conflitto basilare tra pulsione di vita e pulsione di morte ${ }^{56}$ che s'incarnano in proporzioni variabili in ciascuno dei quattro protagonisti; che tale conflitto non vi si manifesti nelle forme usuali dello scam-

53 Il filosofo è esplicitamente richiamato, anche per distinguersene, dallo stesso Freud, Introduzione alla psicoanalisi, in Opere complete, cit., vol. 8, 5594): «Forse scrollerete le spalle: "Questa non è scienza della natura, è filosofia, la filosofia di Schopenhauer". E perché mai, signore e signori, un audace pensatore non dovrebbe aver intuito ciò che una spassionata, faticosa e dettagliata ricerca è in grado di convalidare? E d'altronde, tutto è già stato detto una volta, e molti prima di Schopenhauer hanno detto cose simili. E infine, ciò che diciamo non è neanche l'autentico Schopenhauer. Noi non affermiamo che la morte sia l'unico obiettivo della vita; non trascuriamo la vita, accanto alla morte. Riconosciamo due pulsioni fondamentali e lasciamo a ognuna la propria meta».

54 Ai quali si può aggiungere quello di Thomas Mann, che costituisce anche il collegamento con Freud.

55 All'epoca del Trovatore Verdi non poteva avere alcuna nozione di Schopenhauer, come del resto non l'aveva Wagner, che lo lesse per la prima volta nel 1854. Tuttavia un'edizione del Mondo come volontà e rappresentazione era e dovrebbe tuttora essere presente nella sua biblioteca, insieme a testi di Platone, Pascal e Darwin (vedi L. Magnani, L'ignoranza musicale' di Verdi e la biblioteca di Sant'Agata, in Atti del $I I I^{\circ}$ congresso internazionale di studi verdiani, Milano, Piccola Scala, $12-17$ giugno 1972, Parma, Istituto di studi verdiani, 1974, 250-7, vedi 256), il che è se non altro la traccia di una curiosità intellettuale successiva.

56 Amore e morte è il titolo di uno degli ultimi canti di Leopardi, autore caro sia a Schopenhauer sia a Nietzsche, verosimilmente noto al napoletano Cammarano e a Verdi (un autografo del poeta è conservato nella collezione di S. Agata). Ma era anche il titolo prescelto in un primo momento per quella che sarebbe poi stata La traviata; a parte il fatto che si tratta comunque del sintomo di un interesse per questa tematica, bisogna dire che, se fosse stato confermato, tale titolo avrebbe avuto un significato assai più banale e meno pregnante di quello che ha come sottotitolo occulto del Trovatore. 
bio dialogico o della 'parola scenica', ma nella stessa presenzialità scenica, verbale e musicale dei personaggi ${ }^{57}$ infine, che esso rappresenti il 'contenuto latente' dell'opera, di cui si fa veicolo il 'contenuto manifesto' della vicenda rappresentata. Questa tesi mi sembra adeguata alla concezione dell'opera d'arte come «formazione di compromesso» che consente un «ritorno del represso» - represso dalla censura sociale o ideologico-politica - «reso fruibile per una pluralità sociale di uomini, ma reso innocuo dalla sublimazione e dalla finzione»s. ${ }^{58} \mathrm{E}$ quale idea è più scandalosa, e perciò meritevole di essere censurata, negata, mascherata, di quella che vede l'uomo in balìa di forze impersonali che lo spingono, nell'illusione di appagare i propri desideri ma in realtà agendo al servizio del ciclo della riproduzione, a distruggere e a distruggersi? ?9 $^{59}$ Fra l'altro, questa lettura dà conto dell'impatto emotivo di quella pagina straordinaria che è il Miserere, la cui centralità hanno riconosciuto concordemente critici, quasi mai però ponendosi il problema della sua relazione col sistema testuale dell'intera opera. ${ }^{60}$

Esaminiamo brevemente i quattro personaggi da questa angolatura. (Da quanto ho detto sopra dovrebbe esser chiaro che, anche se per comodità userò espressioni quali «i sentimenti di Azucena», «Manrico è consapevole», non mi riferirò a una inesistente realtà psichica del personaggio, ma alla funzione di questo nel sistema del testo, quale si manifesta in parole da pronunciare, note da cantare, gesti da agire).

57 Per una illustrazione più dettagliata di questa concezione del teatro, musicale e non, rimando a quanto ne ho scritto in «... non senza pazzia», cit., 11-14.

58 Orlando, Per una teoria freudiana della letteratura, cit., 25 e vedi anche 27.

59 «"I bambini non ascoltano volentieri" quando si parla della tendenza innata dell'uomo al 'male', all'aggressione, alla distruzione e perciò anche alla crudeltà. Dio li ha creati a Sua immagine e somiglianza, così che a nessuno piace sentirsi ricordare com'è difficile far coincidere l'esistenza innegabile del male ... con la Sua onnipotenza e suprema bontà» (S. Freud, Il disagio della civiltà, in Opere complete, cit., vol. 10, 5369. Il riferimento è a un verso di una ballata di Goethe).

60 Se lo è posto più chiaramente di tutti Gallarati, Lettura del Trovatore, cit., 130 sg.: «Nelle due scene culminanti, quella d'Azucena, all'inizio del secondo atto, e questa, del 'Miserere', in capo al quarto, le due donne ... esprimono entrambe il loro rapporto con la morte ... Questa è la causa dello sconvolgimento emotivo di entrambe: la morte ha trionfato, o sta per trionfare sull'amore. Ma la presenza della morte, nell'opera, non si limita alle due scene capitali: è continua ... attraversa l'immaginario poetico e drammatico del Trovatore con una presenza sotterranea, meno vistosa ma non meno incisiva di quella del fuoco. È lo sfondo nero su cui rosseggiano le fiamme». 
Azucena è il caso più semplice, e d'altronde l'unico già esplorato dalla critica. I due sentimenti fondamentali che determinano il suo agire, amore figliale e amore materno, producono un conflitto insanabile da cui ella non può liberarsi se non con un reiterato atto distruttivo, la duplice uccisione del 'vero' figlio, che non può non avere anche un esito autodistruttivo. ${ }^{61}$ Ossessione che peraltro, se perlopiù si presenta in forme raccapriccianti, ha anche un aspetto consolatorio, dato che non è difficile capire di qual natura siano «l'antica pace», il «sonno placido» cui anela abbandonarsi tra i suoi monti. Ma, come hanno osservato in molti, e più acutamente di tutti il solito Baldini, Azucena è anche un simbolo universale, una specie di madre natura che somministra imparzialmente vita e morte. ${ }^{62}$

Al contrario, il Conte di Luna appare come una manifestazione della libido allo stato puro, di una pulsione erotica che dà luogo a una sorta di delirio di onnipotenza con tratti infantili («Leonora è mia!») o addirittura blasfemi:

Invano un dio rivale

opponi all'amor mio:

non può nemmeno un dio, donna, rapirti a me! ${ }^{13}$

61 Nell'ultima battuta di El Trovador (ed. cit., 206) Azucena, esclama « YYa estás vengada!» «con un gesto de amargura, y expira». Nel libretto dell'opera (anche nelle sue varie stesure preliminari riportate in $C V C$ ) nulla è detto della sua fine, mentre Verdi prescrisse «cade a piè della finestra». È difficile credere, come pure alcuni opinano, che il Conte, a sua volta distrutto dall'evento, la mandi poi al rogo; ma, una volta compiuta la vendetta, per lei non c'è più una ragione strutturale per continuare ad esistere.

62 Azucena è «compromessa con un gioco cieco e irrazionale degli affetti e del destino e dal nascere e morire, o meglio nell'affiorare e nel perdersi, in un indistinto cerchio di follia». In lei s'incarnano il «delirio del fuoco», «che in modo naturale e imprevisto, scaturisce dalla terra senza ragione: il fuoco della natura, che c’è e deve bruciare; e brucia tendini, nervi, fibre, sentimenti e desiderii, esalta e mortifica, distrugge e ricrea»; e «la tenerezza materna: una tenerezza che non è già più quella di una determinata madre per un determinato figlio, ma che è come uno straziante abbraccio della natura che induce insieme alla diffidenza e all'abbandono» (Baldini, Abitare la battaglia, cit., 250 sg. e 258).

63 Si tratta proprio del Dio cristiano, non del solito «nume» poetico; non meraviglia che su versi come questi si accanisse la censura romana in occasione delle prime rappresentazioni. Vedi anche G. Paduano, Il giro di vite. Percorsi dell'opera lirica, Firenze, La Nuova Italia, 1992, 58. 
Egli è l'unico a non presagire la propria fine, ma anzi a ritenere che l'appagamento amoroso gli conferirà una sorta d'immortalità: «la gioia che m'aspetta, / gioia mortal non è!...» (questo nel libretto; ma chi non avverte la carica libidica di frasi musicali come questa o come «Ah! l'amor, l'amore ond'ardo» farà meglio a occuparsi d'altro). Il suo è però un erotismo frustrato. Il principio di piacere cozza continuamente col principio di realtà («a danno mio rinunzia / le prede sue l'inferno!...»; «In braccio al mio rival!»), e ciò non può non far emergere pulsioni distruttive («Ho le furie nel cor!») che puntualmente - e tipicamente dal punto di vista freudiano - si scaricano in fantasie sadiche:

Ah!... dell'indegno rendere

vorrei peggior la sorte,

fra mille atroci spasimi...

centuplicar sua morte...

Ma è lo sfogo dell'impotenza: la realtà riserba al Conte la più atroce delle disillusioni, tanto più atroce in quanto egli va a infrangersi contro di essa senza averne avuto il benché minimo presagio, al punto che è difficile immaginarlo sopravvissuto all'ecatombe finale. Le sue ultime parole sono una dichiarazione di resa alla morte, tuttavia ancora esorcizzata col nominarla per antifrasi: «E vivo ancor!».

Anche in Manrico la tensione erotica è dominante, sia pure in forma idealizzata come si conviene a un tenore ottocentesco, e prevale nettamente sull'ambizione: l'appagamento amoroso - «s'ei quel cor possiede» - è sufficiente a renderlo «d'ogni re maggior»; ${ }^{64} \mathrm{e}$ anch'egli è convinto che l'amore di Leonora lo renda, lui «mortale», «invitto», se non immortale. Tuttavia sa anche che l'amore è pericolosamente vicino alla morte e che lo sconterà «col sangue [suo]». La prima strofa della 'Romanza' che egli canta nel contesto del Miserere contiene una considerazione universale sulla vita e sulla morte:

Ah che la morte ognora

è tarda nel venir

a chi desia morir!...

64 Seguo qui la lezione del manoscritto di Cammarano (CVC, 205), musicata da Verdi. Il libretto a stampa seguito dall'edizione di riferimento (vedi nota 19) ha: «egli è d'ogni uom maggior». 
È implicita l'idea antitetica che la morte arriva invece troppo presto per chi vorrebbe vivere e amare. Ma di «chi» si sta parlando? La canzone intonata dal Manrique di García Gutiérrez non lascia dubbi:

Despacio viene la muerte, que está sorda a mi clamor; para quien morir desea, despacio viene, por Dios.
È lenta questa morte e sorda al mio dolore: per chi vuole morire è lenta, oh mio Signore, ${ }^{65}$

e il commento di Leonor lo conferma: «Él es; iy desea morir / cuando su vida es mi vida!» («È lui, e desidera morire / quando la sua vita è la mia vita!»); ${ }^{66}$ dunque, almeno dal punto di vista di Leonor, Manrique desidera morire (un desiderio che ella contrappone esplicitamente all'anelito a vivere). Cammarano ha scelto invece una forma impersonale, che non consente di decidere chi sia il soggetto logico dell'enunciato; dunque, egli introdusse nel proprio testo un'ambiguità, mancante nel modello, che lo lascia aperto a due diverse interpretazioni: 1. la morte arriva troppo tardi per chi, come Manrico, desidera morire; 2. la morte arriva troppo presto per chi, come Manrico, vuole vivere e amare. ${ }^{67} \mathrm{Un}$ freudiano ortodosso potrebbe trarne molte illazioni. Io mi limito a osservare che l'ambiguità è confermata dalla musica, dato che Verdi ha intonato le due strofe su una melodia che è al tempo stesso straziante nell'espressione e, per la tonalità maggiore e per la spaziatura dell'accompagnamento, un raggio di luce nel buio del Miserere. Il fatto che si tratti di un classico delle cosiddette melodie da organetto non rende meno vera questa osservazione, anzi la rafforza: Schubert e Mahler hanno molto da insegnare in proposito.

L'ambiguità dell'atteggiamento di Manrico è la sostanza dell'Adagio della sua grande aria, «Ah sì, ben mio, coll'essere». Il cantabile polarizza la tensione tra la gioia per le nozze prossime e il presagio, quasi una certezza, della morte in uno dei più originali percorsi armonici mai attuati da Verdi: Fa minore, La bemolle maggiore, La

65 García Gutiérrez, Il Trovatore, cit., 174 sg. Il dimostrativo «questa», introdotto dalla traduttrice, non è in conflitto col senso della frase.

66 Ibid.

67 Non scioglie l'ambiguità una variante manoscritta di Cammarano: «Tarda è la morte ognora / A chi desia morir!... / O solo mio desir, / Addio Leonora!» (CVC, 211). 
bemolle minore, Fa bemolle maggiore, Re bemolle maggiore. ${ }^{68}$ È notevole che la tonalità minore dell'inizio sia collegata all'immagine delle nozze, mentre i pensieri di morte, pur introdotti e venati da inflessioni in minore, si aprono sempre a tonalità maggiori, dal radioso $\mathrm{Fa}$ bemolle di «ch'io resti fra le vittime / dal ferro ostil trafitto» all'appagato Re bemolle di «e solo in ciel precederti / la morte a me parrà! ${ }^{69}{ }^{9}$

Il personaggio più enigmatico resta Leonora. A lei dobbiamo quella che, espressa nella forma di un luogo comune librettistico, è una definizione adeguata della pulsione di morte quale la concepirà Freud:

Non reggo a colpi tanto funesti...

Oh, quanto meglio saria morir!

L'investimento di energia psichica preteso dal soddisfare la passione amorosa è causa di un disagio così insopportabile che l'annullamento di sé è l'unico modo per sottrarvisi. Luogo comune librettistico, ho detto ${ }^{70}$ si aggiunga che si tratta di una frase assolutamente marginale nel contesto di «Di quella pira», e che, se pure vi si fa caso, la si sente raramente, solo se la cabaletta viene ripetuta. Il fatto è che questa enunciazione non è isolata. Dall'inizio alla fine Leonora sembra dominata da una sorta di furia del dileguare, non fa che parlare della propria morte:

68 In «...non senza pazzia», cit., 15, osservavo che l'analisi melodica di «D'amor sull'ali rosee» ivi contenuta (cap. 6, 111-31) «non aspira a proporre un'interpretazione complessiva del Trovatore, ma potrebbe esserne un tassello, o addirittura la chiave di volta, se solo riuscissi a costruire un'articolazione convincente della struttura drammatica dell'opera. Il presente saggio cerca di soddisfare a tale aspirazione: in effetti anche il cantabile di Manrico, tonalmente 'aperto' come quello di Leonora, «ritrae la tensione fra i due poli dell'ansia e della speranza», laddove «il pendolo emotivo oscilla continuamente tra l'uno e l'altro» (ivi, 127).

69 Bene in proposito Gallarati, Lettura del Trovatore, cit., 110-2. Si può confrontare questo cantabile con quello armonicamente altrettanto ricco di Riccardo in $U n$ ballo in maschera, «Ma se m'è forza perderti»: anche qui il pensiero della morte è onnipresente, ma non mai disgiunto da un profondo senso di disperazione, persino nella chiusa in Do maggiore.

70 Bastino alcuni esempi dello stesso Cammarano, da Lucia di Lammermoor: «Io son tanto sventurata / che la morte è un ben per me»; «Io sperai che a me la vita / tronca avesse il mio spavento»; e da Luisa Miller: «La tomba è un letto sparso di fiori, / in cui del giusto la spoglia dorme: / sol pei colpevoli, tremanti cori / veste la morte orride forme; / ma per due candide alme fedeli / la sua presenza non ha terror...». Si noti, nell'ultimo esempio, l'evidente allusione leopardiana. 
Il mio destin compirsi

non può che a lui dappresso...

S'io non vivrò per esso,

per esso io morirò!

O dolci amiche,

un riso, una speranza, un fior la terra

non ha per me! Degg'io

volgermi a Quei che degli afflitti è solo

conforto, e dopo i penitenti giorni,

può fra gli eletti al mio perduto bene

ricongiungermi un dì.

Tu vedrai che amore in terra

mai non fu del mio più forte;

vinse il fato in aspra guerra,

vincerà la stessa morte.

O col prezzo di mia vita

la tua vita salverò,

o con te per sempre unita

nella tomba io scenderò. ${ }^{71}$

Ora il mio fine impavida, piena di gioia, attendo...

dirgli potrò, morendo:

"salvo sei tu per me!" 72

71 Questo proposito richiama quello di Manrico nei confronti di Azucena: «Madre infelice, corro a salvarti / o teco almeno corro a morir»; ma lì non è prevista la morte del soggetto qualunque delle due alternative si verifichi: madre e figlio saranno entrambi o vivi o morti.

72 Si noti, in questi ultimi due passi, la nettezza dell'antitesi amore/morte, ma anche il paradosso che essa ingenera: l'amore dona la vita, ma solo a costo di sopprimerne un'altra. Come mi fa notare Guido Paduano, al quale sono debitore di questo e di altri illuminanti commenti sul presente saggio, Leonora (come pure, in maniera diversa, Gilda, citata più avanti) si manifesta qui come una variazione del modello classico di Alcesti (si vedano in proposito la sua introduzione a Euripide, Alcesti, Milano, BUR, 2011², 5-40; e, per le realizzazioni musicali del tema, Le due Alceste di Gluck e la drammaturgia classica, in Il giro di vite, cit., 1-28). Mi permetto di riportare qui una sua osservazione: «Il raffronto [di Leonora] con Alcesti può essere un po' più concreto: il rapporto fra linguaggio erotico e linguaggio funerario è dinamico nei due sensi: c'è una dimensione umanistica e ottimistica per cui l'amore "vincerà la stessa morte", e una, se così si può chiamare, decadente, in cui al contrario è la morte che invade 
Prima che d'altri vivere...

io volli tua morir!...

Se per il Conte ho parlato di manifestazioni di sadismo (aggressività che si scarica su un oggetto esterno), in Leonora si riconoscono facilmente tratti di masochismo, dapprima, di nuovo, nella forma attenuata di un luogo comune:

Piombi, ah piombi il tuo furore

sulla rea che t'oltraggiò...

Vibra il ferro in questo core,

che te amar non vuol, non può,

poi resi espliciti in modi ai quali non trovo riscontri nel repertorio librettistico:

Mira, di acerbe lagrime

spargo al tuo piede un rio:

non basta il pianto? svenami,

ti bevi il sangue mio...

calpesta il mio cadavere,

ma salva il trovator!

Strano modo davvero, quello di Leonora, di tentar di salvare il trovatore: prima cerca di placare l'amante respinto dicendogli che non lo amerà mai, poi offre la distruzione del proprio corpo a chi quel corpo desidera freneticamente, per di più motivando l'offerta con l'amore per l'altro. Ciò non può che esasperare la frustrazione del rivale sfortunato, che invece cade anche troppo facilmente nella trappola quando Leonora, cambiando strategia, lo inganna offrendogli il proprio corpo vivo per la vita di Manrico. Si noti la crudezza delle scelte lessicali: «CONTE: Spiegati, qual prezzo? di'. LEONORA (stendendo la destra con dolore): Me stessa! ... che la vittima / fugga, e son tua. ... (M'avrai, ma fredda, esanime / spoglia) $\gg^{73}$.

l'amore, rendendo invivibile la vita del sopravvissuto. Insomma, c'è il buio dentro di noi, ma ci siamo anche noi con la nostra paradossale pretesa di rischiarare il buio» (comunicazione personale del 5 febbraio 2013).

73 Miei i corsivi. In El Trovador Leonor seduce Don Nuño fingendo di amarlo e di voler dimenticare Manrique (ed. cit., 184-9). 
Si può dunque ritenere che l'insieme delle enunciazioni testuali che ho riportato, alcune forse banali altre sicuramente meno, 'faccia sistema' nel delineare il ritratto drammatico di Leonora. Nella realizzazione musicale succede qualcosa di strano: per molte di esse Verdi fa uso di uno stile vocale virtuosistico, brillante e assai difficile, proprio quel tipo di scrittura che ha fatto vedere il personaggio, e addirittura l'intera opera, come uno sguardo retrospettivo a un mondo stilistico che egli aveva ormai abbandonato. Questo modo di cantare celebra il suo ultimo trionfo nella cabaletta di Violetta «Sempre libera degg'io», una pagina concepita contemporaneamente alla composizione del Trovatore. ${ }^{74}$ Nella Traviata, però, l'uso - in questa sola pagina - del virtuosismo vocale è d'interpretazione univoca: dà espressione al desiderio di Violetta di godere fino in fondo il breve tratto di vita che le resta da percorrere: ella sfiora la vertigine del «di voluttà ne' vortici perir!...» solo per esorcizzare la triste certezza di dover morire per consunzione. È azzardato ipotizzare che nel Trovatore Verdi abbia impiegato estensivamente lo stesso mezzo stilistico per rappresentare il cupio dissolvi di Leonora, la sua vertiginosa corsa all'abisso? All'interno del quadro teorico in cui ho collocato questa lettura l'ipotesi è accettabile, e tuttavia se ne affaccia un'altra che mi sembra compatibile con la precedente.

Se è vera l'idea che l'opera d'arte realizza un ritorno di contenuti repressi perché socialmente riprovati, bisogna dire che, negli esempi classici sui quali si è esercitata la teoria, tali contenuti sono «resi innocui dalla sublimazione e dalla finzione» per mezzo di un raffinato sistema di metafore, reticenze, allusioni. Nel libretto del Trovatore, almeno per quanto riguarda Leonora, i contenuti inquietanti sono invece, come abbiamo visto, anche troppo espliciti; ${ }^{75}$ nel 1853 essi furono infatti sistematicamente occultati dalla censura romana. ${ }^{76} \mathrm{Ma}$ come funziona la cosa per gli innumerevoli spettatori che da allora hanno visto e ascoltato Il trovatore e ne hanno letto il libretto intatto? Un primo livello di

74 Non considero qui l'impiego che Verdi farà del canto di coloratura per le parti di Hélène nelle Vêpres siciliennes e di Eboli nel Don Carlos, e anche di Oscar in Un ballo in maschera, legato a questioni di genere in una tradizione diversa da quella italiana.

75 Un altro libretto di Cammarano, la già citata Maria de Rudenz, è ancor più crudo del Trovatore nel presentare un intero repertorio di passioni distruttive; e, forse proprio a causa di tale esplicita crudezza, il risultato artistico è meno convincente.

76 Si vedano i numerosi interventi documentati dall'edizione critica (cit. alla nota 19). 
attenuazione è dato, direi, dallo stesso statuto letterario del genere librettistico nell'Ottocento. ${ }^{77}$ Non c'è solo l'oscurità, voluta o meno, degli accadimenti, non c'è solo il filtro del linguaggio aulico e iperletterario: c'è anche il fatto che la maggior parte degli spettatori legge il libretto per capire, più o meno, cosa succede sulla scena, non per cogliere parola per parola il significato di ciò che vi si dice; per questo Verdi elaborò, per i discorsi veramente importanti, il concetto di 'parola scenica', ${ }^{78}$ di cui proprio nel Trovatore si trovano scarsissime tracce. Lo stesso vale per lettori più scaltriti quali dovrebbero essere i critici: pochissimi prendono sul serio il testo librettistico come veicolo di contenuti più profondi di quelli immediati (un po' come accade coi motti di spirito analizzati da Freud). Di conseguenza si è propensi a non vederli anche quando, ed è il caso di Leonora, essi sono per così dire squadernati sotto i nostri occhi; tanto è vero, che finora nessuno ci ha fatto caso.

Il secondo filtro è ovviamente quello interposto dalla musica. Se di norma solo una parte delle parole cantate giunge distintamente alla percezione dello spettatore (persino in Monteverdi, in Lully e in Pizzetti), tanto minore sarà il tasso di comprensibilità quanto più il compositore farà uso di una vocalità belcantistica. Ed è appunto quanto accade nel caso di Leonora: il suo stile vocale prevalente è la negazione (in senso freudiano) del contenuto censurabile di ciò che sta dicendo. ${ }^{79}$

Non è però solo questione di cogliere il senso delle singole parole e frasi. Cerco di essere più preciso citando di nuovo Orlando:

Dire con Lacan che l'inconscio è il regno del significante, equivale in concreto a dire che nei linguaggi dell'inconscio non si dànno mai significanti trasparenti perché non si dànno mai significati scoperti: dunque il significato è

$77 \mathrm{Ma}$ in realtà di qualsiasi genere letterario: «quand'anche non ci sia la negazione freudiana del ritorno del represso a scindere il soggetto o la coscienza, esso resta neutralizzato praticamente dalla portata fittizia dell'istituzione letteraria, che funge già da negazione freudiana in quanto tale» (Orlando, Per una teoria freudiana della letteratura, cit., 87 sg.).

78 Vedi Della Seta, «...non senza pazzia», cit., 203-25.

79 Non sempre, peraltro. In alcuni casi l'intonazione musicale consente una nitida percezione delle parole e ne esprime adeguatamente il contenuto: «svenami, / ti bevi il sangue mio ... / calpesta il mio cadavere». 
sempre invisibile e solo il significante è visibile. Dire con la retorica recente che un linguaggio verbale è opaco quando attira l'attenzione su se stesso coprendosi di figure, anziché restare impercettibile e lasciar trasparire immediatamente il significato, equivale a indicare una somiglianza coi linguaggi dell'inconscio: cioè con quelli la cui opacità per eccellenza è tanto fitta da farsi alla lettera oscurità. ${ }^{80}$

Come ho già detto, il linguaggio librettistico è di per sé connotato da un alto tasso di figuralità, che sortisce l'effetto, voluto o meno, di renderlo «opaco», oscuro, come vuole la vulgata critica. Non bisogna però dimenticare che, fin dal XV secolo, col termine musica figurata o figuralis si designava un tipo di melodia fiorita e ornata, e 'figurazioni' si chiamano ancor oggi le componenti di una linea melodica siffatta; diverso, rispetto all'uso letterario del termine, è il percorso semantico a partire dal latino figura, ma comune è la matrice retorica dell'uso nei due ambiti. ${ }^{81}$ Affermare che le figurazioni melodiche hanno qualcosa in comune con le figure del testo non è un gioco di parole; piuttosto, evidenzia il fatto che la funzione comune ai due procedimenti è quella per cui il linguaggio in opera «attira l'attenzione su se stesso»:

la figura come alterazione del rapporto di trasparenza tra significante e significato ha la sua origine nei linguaggi asociali e funzionalmente non comunicanti dell'inconscio. Là essa si addensa a piacere, e domina al punto da dissimulare se stessa dissimulando il senso che tuttavia per compromesso esprime. In un linguaggio comunicante e sorvegliato dell'io cosciente la figura non potrebbe prodursi che come disturbo ed errore, se per assurdo l'uomo fosse un animale privo di inconscio e avesse come prerogative innate una traspa-

80 Orlando, Per una teoria freudiana della letteratura, cit., 60.

81 L'aggettivo figurata si applicava in origine (sec. XIII) alla musica misurata (polifonica) in quanto i valori di durata vi erano rappresentati da figurae geometriche (quadrato, losanga); nello stesso periodo si usava normalmente il termine colores, mutuato dalla retorica, per designare ogni sorta di artifizi compositivi atti a ornare il discorso musicale. Successivamente figuratus e i suoi corrispettivi nelle principali lingue europee vennero a significare «Florid, i.e. elaborated with various kinds of musical artifice. At its broadest 'figural music' simply means polyphonic or concerted music, as opposed to plainchant. All the terms could be applied to a single part, e.g. to distinguish a decorated line or cantus figuratus from the plainchant or cantus planus to which it might be added as a descant» (M. Tilmouth, voce Figural, figurate, figured, in Grove Music Online [http://www.oxfordmusiconline.com/subscriber/article/grove/music/ 09620]). 
renza assoluta nell'uso della parola e una logica rigorosa nell'uso del pensiero. L'una e l'altra essendo invece radicalmente per l'uomo conquiste e insieme costrizioni, contro queste costrizioni il compiacersi della figura prende il valore di un «ritorno del represso formale». In altri termini la figura assume come nuova funzione, socialmente istituzionale, un compromesso orientato all'inverso e un piacere trasmissibile ad altri. ${ }^{82}$

Con questa funzione, il canto 'figurato' era impiegato estensivamente in quel tipo di prodotto artistico per eccellenza allusivo e reticente che era l'opera seria settecentesca, e con altrettanta sicurezza Verdi sembra averlo fatto rivivere a questo fine, un'ultima volta, nel Trovatore. Con ciò acquista un senso preciso l'intuizione di quel critico finissimo che fu Luigi Baldacci, quando osservava che la linea interpretativa di Mila (cui ho accennato più sopra) «abbisogna a volte di integrazioni che recuperino alla vita musicale personaggi, come la Leonora del Trovatore, indebitamente relegati nel dominio del bel canto, laddove quel canto è dramma oscuro dell'Es non meno di quello di Azucena». ${ }^{83}$

Se quanto ho detto finora è accettabile, dobbiamo ripensare a fondo la posizione comunemente attribuita al Trovatore nel percorso creativo di Verdi. Non che rappresentare il commiato dalla tradizione rossiniana, proprio in virtù dell'impiego sistematico di forme e di stili vocali desueti quest'opera apre una nuova fase di tale percorso, in cui la prospettiva ideologica degli anni giovanili viene ribaltata. Fino a qui le eroine e gli eroi verdiani sono espressioni positive dell'eros: lottano strenuamente per affermarlo, soccombono dimostrando un disperato attaccamento alla vita, che lasciano con rimpianto come Gilda («Ah! presso alla morte, / sì giovine, sono»), come Violetta («Gran Dio! morir sì giovane, / io che penato ho tanto»); ancora nel Ballo in maschera Amelia, se è sfiorata dalla tentazione dell'oblio, sa pure che nulla resterebbe al suo «povero cor» una volta «perduto l'amor» (e Riccardo è pronto a ricordarglielo: «non sai tu che di te resteria / se cessasse di battere il cor!»). Ma già Simone Boccanegra rimpiangeva di non aver trovato ancor giovane la propria tomba nell'alveo materno del mare, così come Aida prefigurerà per sé «pace forse, e oblio» nei «cupi vortici»

82 Orlando, Per una teoria freudiana della letteratura, cit., 64.

83 L. Baldacci, I libretti di Verdi, in: Il melodramma italiano dell'Ottocento. Studi e ricerche per Massimo Mila, Torino, Einaudi, 1977, 113-23, la citazione a 119. 
del Nilo. ${ }^{84}$ «Pace, mio Dio», invoca la Leonora della Forza del destino, e immediatamente fa chiaro di quale pace stia parlando: «Dio!, fa' ch'io muoia». Tre dei cinque protagonisti del Don Carlos - quattro col frate/Carlo V - non vedono che nella tomba una speranza di sonno e di pace. Infine, nelle ultime battute di Otello si realizza l'identificazione inquietante tra appagamento erotico e dissoluzione del Sé. Qui, davvero, il non detto è più del detto, ${ }^{85} \mathrm{ma}$ siamo in un'altra epoca, in compagnia di un altro poeta e forse anche di un altro musicista. Come che sia, mi sembra che quella dimensione oscura che il Verdi maturo e tardo esplorò con la sua ineguagliabile capacità di penetrazione del mondo interiore dell'uomo, nel Trovatore egli la seppe presentare quasi allo stato puro, scevro di ogni psicologismo, in forme così depurate da ingenerare l'immagine di un'opera quant'altre mai astratta e 'metafisica'.

Un'ultima considerazione. Come ho più volte avvertito, la mia lettura si basa interamente sul testo drammatico-musicale dell'opera, al massimo con qualche appiglio in testi contigui quali le tracce del processo creativo e il modello letterario del libretto. Siamo troppo scaltriti per fare appello all'autore e alle sue intenzioni; tanto più per pretendere 'fatti' che possano «dar forma di prova» agli indizi su cui costruiamo le nostre interpretazioni. Eppure, se siamo in possesso di qualche dato collegabile al vissuto dell'autore empirico, dobbiamo davvero vietarci, non dico di considerarlo una prova, ma almeno di assumerlo come un ulteriore frammento di testo utile a orientare l'interpretazione in un senso piuttosto che in un altro? Dieci giorni dopo l'andata in scena del Trovatore, il 29 gennaio 1853, Verdi scrive all'amica Clara Maffei:

Dicono che quest'Opera sia troppo triste e che vi sieno troppe morti ma infine nella vita tutto è morte! Cosa esiste?... ${ }^{87}$

84 Vedi anche Radamès: «d'ogni gaudio / la fonte inaridita, / sol bramo di morir».

$85 \mathrm{Ma}$ in realtà era detto esplicitamente alla fine del primo atto: «Venga la morte e mi colga nell'estasi / di questo amplesso / il momento supremo!». Vedi Paduano, Il giro di vite, cit., 151-4, e anche, in sintesi, TuttoVerdi, cit., 153.

86 Il richiamo a Il trovatore (1917) di Giorgio De Chirico, «uno dei più celebri tra i suoi manichini metafisici», è in Mila, La giovinezza di Verdi, cit., 448.

87 Cit. da Giuseppe Verdi, Lettere, a cura di Eduardo Rescigno, Torino, Einaudi, 2012, 282. 